\title{
Integrating Lecture and Laboratory Work for a Materials Chemistry Course to Engage and Motivate Students through Highly Visual and Intriguing Syntheses
}

Karine Molvingera,*, Rose-Marie Ayrala, Jean-Sébastien Filhola,b

a ICGM, Univ Montpellier, CNRS, ENSCM, Montpellier, France

b Faculté des Sciences, Univ Montpellier, Montpellier, France

\section{ABSTRACT}

An original integrated course called "Chemistry: Magical Science" at Montpellier University (France) is presented. This approach mixes tutorials and practical work by using the pretext of high-tech materials synthesis to teach students knowledge, skills, and practical techniques. It also educates students about chemical risks, bibliographic research and trains them for oral presentations. The practical work is partly directed and partly autonomous. This work involves a description of this course and its evaluation.

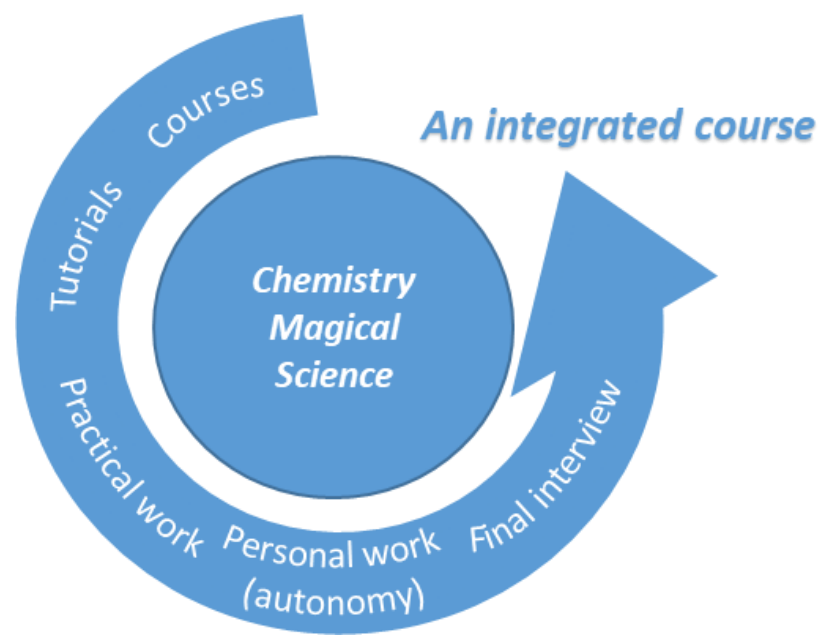

\section{KEYWORDS}

First-Year Undergraduate/General, Second-Year Undergraduate, Hands-On Learning/Manipulatives, Materials Science, Student-Centered Learning, Synthesis, Undergraduate Research.

\section{INTRODUCTION}


Some of the objectives of science education at university level is to impart a global knowledge of science and technology, and also to train future engineers and scientists. For this, science teaching at university is usually composed of theoretical courses and practical laboratory work. At undergraduate level, a classical course organization alternates lectures and practical work throughout the semesters. Unfortunately, these two types of teaching are often quite distinct, with evaluation of specific skills, different teachers and a chronology (the sequence of lectures and practical work) that is not necessarily optimized, for reasons of organization. Furthermore, when students are actively involved in the construction of their knowledge base, they tend to learn better: ${ }^{1}$ in order for a student to invest time learning chemistry in a meaningful way, knowledge cannot simply be passed verbally from teacher to student. Therefore, students should be active participants in the learning process so that they might construct their knowledge of a subject through an interacting combination of theory and practice.

This is particularly so in the case of chemistry, which is an experimental science, since laboratory work plays a key role in the chemist training. Nevertheless, recent researches in the teaching of experimental chemistry in higher education remain rare: Obenland et al. ${ }^{2}$ reviewed the history of lab teaching and showed that it is still quite controversial. ${ }^{3,4}$ Some have argued that experimental work should be removed. Obenland et al. point out that when taught as a traditional laboratory course, experiments do not encourage students to deepen their understanding of chemical concepts. They propose that students think about chemistry by discussing data, and gaining an understanding of concepts rather than by following protocols. ${ }^{2}$ For many university professors, teaching chemistry without practical work is not conceivable since the laboratory is by definition the place where students are confronted with the reality of the chemical world and make connections with models and theories. $5,6,7$

Hofstein et al. point out that the studies conducted so far have not highlighted simple relationships between experimental practice and student learning. ${ }^{8}$ However, a recent study in Australia and the United Kingdom shows that there is a general consensus on the need to develop transferable practical skills while transmitting theoretical knowledge. ${ }^{9}$ The underlying assumption in each of these studies is 
that students will be better at learning chemistry ${ }^{4}$ only if they perceive the subject in a positive way and come to understand and internalize knowledge.

Furthermore, many authors have pointed out the increased enthusiasm of the students that are interested and motivated by experimental courses. For example, Furlan et al. 10 show that nanomaterials based experiments "allowed students to gain exposure to the modern field of nanoscience and technology and to gain direct working experience with functional nanocomposite materials". Porter ${ }^{11}$ shows an example of courses on nanotechnology where students participate in lecture and guided discussion, in writing and laboratory exercises. Other examples of courses based on materials science are described in the literature. ${ }^{12}$ Since more than 40 years, ${ }^{13}$ different approaches including the term "integrated" have been undertaken in order to improve undergraduate chemistry education. This term can have different meanings such as laboratory course in the same physical space ${ }^{14}$ or a program of study combining sub-disciplines of chemistry into a single laboratory sequence. ${ }^{15}$ For the first example, we can notice the work of Budner et al. ${ }^{14}$. Their approach includes moving away from a traditional lecture/lab separation, into an integrated lecture/lab course model incorporating problem-based learning. They offer laboratory works on general chemistry concepts with a reduced guidance after a few courses. According to the authors, students are more engaged, appreciate the combined lecture and lab but unfortunately no "dramatic increase in assessments scores" is observed. The second approach can be illustrated by the recent article of Monga et al. ${ }^{15}$. They show the implementation of an integrated approach to upper-level undergraduate chemistry laboratory instruction, incorporating student choice both in the selection and sequencing of the experimental work. The approach involves combining laboratory work in several traditional subdisciplinary areas of chemistry in a single course. The assessment of this new program is based on the students statements. The authors notice that this new curriculum reveals several strengths and weaknesses.

Our approach is quite different. By the term "integrated", we mean to integrate training on security and bibliography to a series of laboratory works that give more and more autonomy and freedom to students up to the point at the end of the course to improve/design a new laboratory synthesis taking into account all factors involved in the safe development of a lab work. For each lab 
experiment, a summary of courses on specific chemistry topics are given by the teacher in the laboratory space. Furthermore, in our module, laboratory works are based on the synthesis of original materials.

"Chemistry: Magical Science" (CMS) is an original laboratory class taught at the University of Montpellier (France) to first and second years undergraduate students. "Chemistry: Magical Science"16 is used as a pretext for the syntheses of high-tech materials to train students into multiple types of knowledge and skills. Students learn technical skills (use of precision scales, dosage, synthesis, etc), associated with chemical risks and safety management, and general skills (use of scientific and technical literature, oral presentations etc). The teaching of this course is not subdivided in discreet, independent lectures, tutorials, practical work, but on the contrary, is a completely integrated training course. For this, the teaching team uses or has specifically developed advanced syntheses of materials with remarkable properties that can be straightforwardly tested such as superconductors, photonic crystals, ferrofluids, precious stones (rubies, sapphires) or long-lasting phosphors. Most materials synthesis in "Chemistry: Magical Science" are recent and adapted or specifically developed for the course. The synthesis of materials with "magic"-like properties is thus used as a bait. This awakens desire to make these materials and invokes the students' curiosity about the physical reasons for these "amazing" properties. The teaching of "Chemistry: Magical Science" tries to push students towards the research world: the ultimate goal is to encourage interest in scientific research. However, CMS uses practical work as a tool for creating knowledge. The syntheses are thus the starting point for a completely integrated course for students, which allows them to quickly gain autonomy in developing valuable skills. This type of approach has been encountered in the literature in other fields of chemistry. ${ }^{17,18}$

Therefore, in this article, we present the course and its mode of evaluation. These two points can be formulated as follows:

1) How does one devise an integrated teaching course based on a practical laboratory approach?

2) Is this integrated approach more effective qualitatively (for example, students' perceptions), but also quantitatively, in the transmission of knowledge? 


\section{CHEMISTRY: MAGICAL SCIENCE}

Course overview

We represent this module by the scheme in Figure 1 (More information in the Supporting Information).
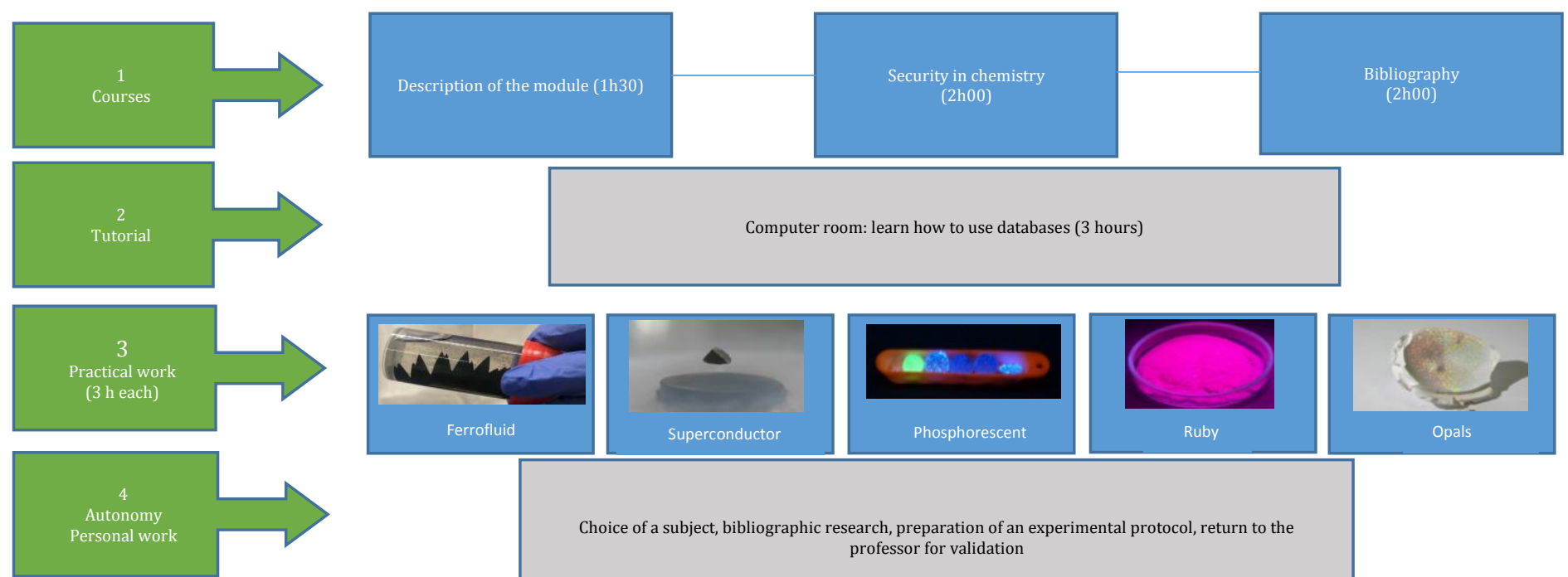

Ferrofluid

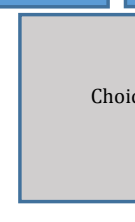

Choice of a subject, bibliographic research, preparation of an experimental protocol, return to the professor for validation
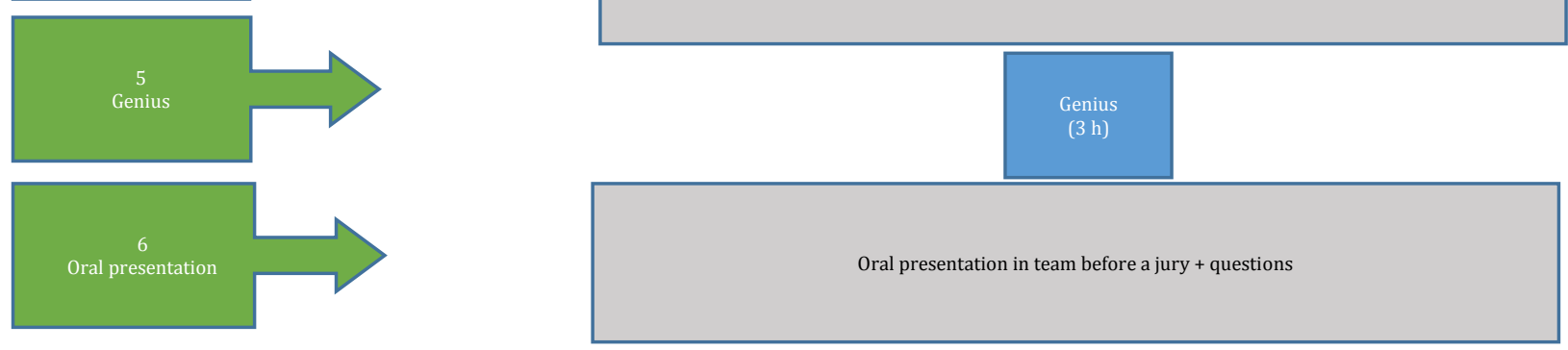

Figure 1. Scheme of the organization of the module of "Chemistry Magical Science"

In the first course (week 1), the organization of CMS module is presented to the students (including the schedule with the different lectures, tutorials and practical work sessions). The students are introduced for the first time to transferable skills such as how to read a scientific article, basic oral communication, teamwork and presentation skills. The organization of the practical work is then presented (including the fact that they will work in groups of three with a team leader appointed at 
each laboratory session), and the criteria for evaluation (bibliography report, laboratory notebooks and a presentation of their laboratory work called "Genius") are given. The materials to be synthesized during the practical sessions are presented (superconductors, phosphorescents, opals, ferrofluids ...) as well as the advanced synthesis methods (sol-gel/microwave/high temperature/biphasic polymerization syntheses).

The second session (week 2) is about laboratory safety. The two-hour class focuses on the behavior that a student in a laboratory should adopt (personal protection, compliance with safety rules, etc.), the risks associated with the use of chemicals, good practice in waste disposal and also how to react in the event of an accident.

The third session (week 3) is devoted to bibliographic research. The databases and their use are presented to them. A tutorial session (week 4) allows them to query the general (google...) and scientific (web of science....) databases about one of the topics covered in the practical work (superconductor, ferrofluid ...). It will then be evaluated.

Then five laboratory sessions (3 hours each, weeks 5-9) follow including the synthesis of ferrofluids, superconductors, phosphorescents, rubies and opals. Part of the protocols materials have been published in the Journal of Chemical Education. ${ }^{19,20,21}$ Table 1 contains a brief description of the synthesis of each of the materials (See the Supporting Information also). These laboratory sessions were chosen to illustrate key concepts, to see things for real, to search for the relevant synthesis protocol in the literature, to introduce new equipment or methodologies to the students, to train them in specific practical skills, to manage safety issues, to teach experimental design and finally, to develop collaborative, logical reasoning and interpretative skills. ${ }^{6}$

Table 1. Brief Methods for the Synthesis of the Materials in the Course Chemistry: Magical Science

Materials Brief Description of the Synthesis

Ferrofluids /

Ferrogel $a$

Superconductor
The synthesis of magnetite nanoparticles is done using a mix of $1 / 3 \mathrm{Fe}$ (II) and $2 / 3 \mathrm{Fe}$ (III) with a dropwise addition of an $\mathrm{NH}_{3}$ solution. The magnetic nanoparticles are then washed, followed by a sequence of magnetic decantation and elimination of the nonmagnetic phase. The ferrofluid is obtained by magnetic decantation and drying followed by addition of a few drops of a solution of tetrabutylammonium hydroxide as a dispersant. The ferrogel is obtained by dispersion of the nanoparticles in a PVA solution that is jellified using borax. Magnetic properties are tested using a strong magnet.

A microwave-assisted sol-gel synthesis of $\mathrm{YBa}_{2} \mathrm{CuO}_{3} \mathrm{O}_{7-\mathrm{y}}$ is performed. The precursors used are nitrates $\mathrm{Y}\left(\mathrm{NO}_{3}\right)_{3}, 5 \mathrm{H}_{2} \mathrm{O}, \mathrm{Ba}\left(\mathrm{NO}_{3}\right)_{2}$, and $\mathrm{Cu}\left(\mathrm{NO}_{3}\right)_{2}, 3 \mathrm{H}_{2} \mathrm{O}$. After addition of complexing anions, the modification of the $\mathrm{pH}$ by addition of ammonia allows a shift in the hydrolysis 
and condensation balances. The phase $\mathrm{YBa}_{2} \mathrm{Cu}_{3} \mathrm{O}_{7-\mathrm{x}}$ is obtained by drying and then annealing the gel at high temperature. The superconductivity of the pellet is checked by small magnet levitation at liquid nitrogen temperature.

Phosphorescent ${ }^{b}$ The material is synthesized from a solution of the different metal nitrates (europium, dysprosium, aluminium, and strontium) in a small volume of deionized water. Then, urea is added to the solution and stirred until the urea is completely dissolved.

The powder is crushed and washed using the Büchner funnel and the sidearm flask under vacuum with both distilled water and ethanol. The solid is dried at $100{ }^{\circ} \mathrm{C}$ in a drying oven. The resulting white powder is tested for fluorescence and phosphorescence under UV light. A strong green fluorescence and long-lived green phosphorescence are observed.

The synthesis was adapted to produce $\mathrm{CaAl}_{2} \mathrm{O}_{4}: \mathrm{Eu}$ :Dy, by replacing the strontium nitrate by calcium nitrate tetrahydrate, $\mathrm{Ca}\left(\mathrm{NO}_{3}\right)_{2} \cdot 4 \mathrm{H}_{2} \mathrm{O}$. The doped aluminate displays a blue fluorescence and blue phosphorescence.

Rubies $^{c} \quad$ Rubies are synthesized by microwave-assisted, self-propagating combustion of a gel formed from aluminium and chromium nitrate with urea and treated similarly to the phosphorescent synthesis. The formed nano-rubies can be checked by observation of a strong red fluorescence upon exposure to UV light $(380 \mathrm{~nm})$. Rubies can then be grown to macroscopic size up to 0.5 carats by flux synthesis.

Opals A synthesis of nanoparticles of defined size is carried out by two-phase polymerization. For this purpose, temperature and agitation conditions are very important.

Opals are obtained by polymerization reaction of methyl methacrylate MMA in an inert atmosphere, initiated by a radical initiator (2,2-azobis(2-methylpropionamide)). After centrifugation, the solid obtained is iridescent. The nano size can be checked directly by observation with a SEM.

aSee ref 19. ${ }^{b}$ See ref 20. ${ }^{c}$ See ref 21.

A specific session called "Genius" is then carried out (week 10). Before this session, students are asked to choose between several possible themes (e.g. macroscopic sapphire synthesis, superhydrophobic synthesis, silver nanoparticles synthesis...). They prepare a protocol of synthesis in their own time by doing bibliographic research and taking into account chemical risks and by checking the availability of chemicals and the equipment needed with the teacher or laboratory technicians. Classic examples include the preparation of magnetic nanocomposites 10,22, nickel nanowires ${ }^{23}$, gold and silver ${ }^{24}$ nanoparticles, and quantum dots ${ }^{25,26}$. All of these syntheses can be adapted to practical work for the Genius session. The students remain in contact with the different teachers that orientate the searched and validate the protocols (in particular to prevent safety issues).

The "Genius session" lasts 3 hours and the proposed synthesis is conducted.

Finally, an oral preparation session is proposed (week 11). The instructions for their oral presentation are given to them. They present their "Genius" in groups of three or four and the speech must be balanced between each student whether it is for the presentation or for the questions. The structure to make an oral presentation is provided (objective, methods, results, conclusion without 
forgetting to quote bibliographical references). Instructions on the slides (layout, animation, colors, text ...) as well as their speech (voice, gestures, respect the time, capture the attention ...) are given to them. The evaluation grid is presented to them and instructions on the dress code are also given (suits, ties and formal clothes welcome). A detailed description of good practices in oral presentations is done in direct link with future job interviews.

Students are evaluated on a final presentation of their "Genius" session, their laboratory notebook and the bibliography report. For the final presentation (week 12), the jury is mainly composed of people external to the CMS teaching team. The examination consists of a 15-minute presentation followed by a 15-minute discussion with the jury. This is a first experience of the presentation of scientific results (in the same manner as a Master's or PhD thesis defence or at conferences).

\section{Methodology}

The "Chemistry: magical science" class is open to all first and second year students at the University of Montpellier. Nevertheless, each class has a limited size and only the first 22-28 students that apply on the local online application system are selected on a first come first serve basis. The total number of applications ranges between 100 and 200 each semester. The class is not specifically advertised to the students, as it is already in high demand, leaving those not selected frustrated. To ensure that our following statistical results are not biased (the selection process may suggest that the best students register first), we checked their scores before they registered for the course.

For first-year students, not all the students enrolled are chemists, half of them are in mathematical, physical or geological fields. They are not specialists in chemistry: "Chemistry: Magical Science" module is open to all scientific students and not only to chemists. For the other half of firstyear students, their first semester mark (before CMS) is slightly higher than the other students in chemistry (average of 10.5/20 compared to $9 / 20$ for the whole). Overall, first-year students do not represent a group of students with better results in chemistry.

For the second-year students, the total number of chemistry students has an average of 8.7/20, those who have followed the "Chemistry: Magical Science" course have an average of 8.9/20, so roughly equivalent. The data show that we have a reasonable random sampling of students. 
All courses and laboratory sessions were filmed. During the safety and bibliography courses, the camera focused on the teacher and the slides. For laboratory sessions, the camera focused on the teacher when he explained certain practical and theoretical points. The rest of the time of the laboratory work, it was focused on different groups of students when they were manipulating. At the end of the laboratory work, we were also able to ask them about their feelings.

For this manuscript, two kinds of student populations have been studied through surveys:

- Firstly, two groups of students from the "Chemistry: magical science" course itself: 22 first year students and 23 second year students (this includes a survey of student perception of the course):

All of these students select this course for its attractive, interesting side, to have the opportunity to learn the synthesis of original materials, to learn experimental techniques and to acquire knowledge. In terms of the surveys, the students are solicited just after having realized the series of practical sessions as part of the "Chemistry: magical science" course. Their feelings about this practical work are therefore quite significant.

- Secondly, a survey is given to third year students in chemistry (147 people) at the beginning of the academic year, some of them have followed "Chemistry: magical science" in previous years (numbering 78 - the CMS team) and some have not (numbering 69 - the control team). It is important to note that all of the students have followed the same chemistry degree courses during the previous two years. All of the courses are mandatory, except for a few optional modules such as CMS. However, only the "Chemistry: magical science" covers the concepts of bibliography, safety in chemistry and laboratory work. Therefore, the only difference between the two samples is the follow-up of this option by the students. Thus, this survey allows us to evaluate the effects of the module in terms of knowledge in security, bibliography, and motivation. In addition, at the end of their third year, these students carry out a tutored project. We also looked at the influence of CMS on these projects.

\section{RESULTS AND DISCUSSION}

Analysis of the survey of feelings

During these different sequences of practical work, the authors (KM and RMA) served as observers and in no case participated in the teaching of the various subjects, which was managed by JSF. However, during the sessions, discussion with students was possible. Students could express their 
opinions on the course. The majority of the students found the class useful and believed that it taught them new and valuable skills that they felt would help them in their chemistry education. ${ }^{17}$

Then, to assess the course from the point of view of the students, a small survey was carried out at the end of the module. Students were asked about their experience of "Chemistry: Magical Science" laboratory sessions.

The results are given in Table 2, where the answers from both populations (first year + second year) for their perception of the "Chemistry: Magical Science" class are shown.

\section{Table 2. Comparative Results of the First- and Second-Year Student Survey Items on Experience Perceptions}

Statements for Response

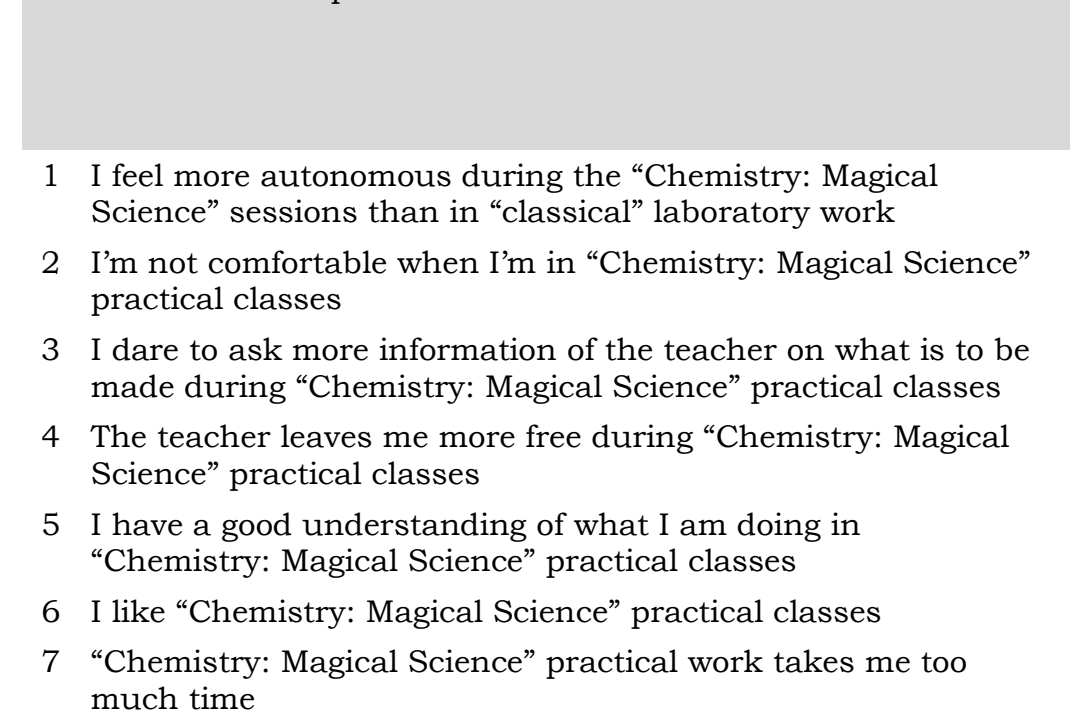

\begin{tabular}{|c|c|c|c|c|c|c|c|}
\hline \multicolumn{8}{|c|}{ Distribution of Responses by Category, $N=45$} \\
\hline \multicolumn{2}{|c|}{$\begin{array}{c}\text { Strongly } \\
\text { Agree }\end{array}$} & \multicolumn{2}{|c|}{$\begin{array}{l}\text { Rather } \\
\text { Agree }\end{array}$} & \multicolumn{2}{|c|}{$\begin{array}{l}\text { Rather } \\
\text { Disagree }\end{array}$} & \multicolumn{2}{|c|}{$\begin{array}{l}\text { Strongly } \\
\text { Disagree }\end{array}$} \\
\hline$N$ & $\%$ & $N$ & $\%$ & $N$ & $\%$ & $N$ & $\%$ \\
\hline 21 & 47 & 16 & 36 & 6 & 13 & 2 & 4 \\
\hline 3 & 7 & 6 & 13 & 6 & 13 & 30 & 67 \\
\hline 22 & 49 & 18 & 40 & 3 & 7 & 2 & 4 \\
\hline 22 & 49 & 16 & 36 & 5 & 11 & 2 & 4 \\
\hline 15 & 34 & 22 & 49 & 6 & 13 & 2 & 4 \\
\hline 34 & 76 & 8 & 18 & 2 & 4 & 1 & 2 \\
\hline 3 & 7 & 8 & 18 & 16 & 35 & 18 & 40 \\
\hline
\end{tabular}

The students' reactions to practical work are often positive. Firstly, they seem to appreciate "Chemistry: Magical Science". 42 out of 45 students like this module. They feel autonomous (37 out of 45), and free (38 out of 45). They seem comfortable during practical work (36 out of 45). Students dare to ask the teachers questions (40 out of 45). They have a good understanding (37 out of 45). For 34 out of 45 students, the practical work does not take too much time.

At the end of the laboratory work, we asked the students how they experienced this laboratory work. These comments are consistent with their answers to the questionnaire. After transcribing their words, we see that the students feel involved in this module, which is much more interesting for them ("These practical works are more interesting and more understandable than the ones we did last year."). 
Firstly, their interest stems from the opportunity to synthesize materials that have remarkable properties and have applications in everyday life ("The materials are impressive, especially when we see our superconductor levitating"). Secondly, the organization of the module allows them to learn many more things: the course on safety followed by practical work allows them to be more aware of the risks in situation during the lab, and the course on bibliography allows them to become autonomous in their research for preparing the Genius session.

Through this survey, we examined different points (motivation (Table 3), bibliography (Table 4), and security/safety (Table 5)) that can be improved by this way of teaching. The results of this study compare two populations (among third-year students), one of which followed the CMS module in previous years (78 people - the CMS group) and the other populated by those who did not (69 people, the control group). This is the only difference between the two populations.

- Regarding motivation:

Questions about their future are asked to find out if there is a difference between the two populations in terms of their motivation to continue studying. In other words, are students who have followed CMS more motivated to study for a long time?

\begin{tabular}{|c|c|c|c|c|}
\hline \multirow{3}{*}{$\begin{array}{l}\text { Statements for } \\
\text { Response }\end{array}$} & \multicolumn{4}{|c|}{ Students Answering "Yes" } \\
\hline & \multicolumn{2}{|c|}{ CMS Group $a$} & \multicolumn{2}{|c|}{ Control Group } \\
\hline & $N$ & $\%$ & $N$ & $\%$ \\
\hline $\begin{array}{l}1 \text { Would you like to } \\
\text { continue your } \\
\text { studies in a } \\
\text { Master's degree? }\end{array}$ & 76 & 97.5 & 57 & 82.5 \\
\hline $\begin{array}{l}2 \text { Do you plan to } \\
\text { prepare a Ph.D. } \\
\text { thesis in } \\
\text { chemistry? }\end{array}$ & 15 & 19.0 & 11 & 16.0 \\
\hline$a N=78 . b^{b} N=69$. & & & & \\
\hline
\end{tabular}

More students who have followed the "Chemistry: magical science" module are willing to continue to a Master's degree. This result is statistically significant with a $\chi^{2}=9.4$. The second question concerning a potential $\mathrm{PhD}$ thesis thereafter, is not statistically significant $\left(\chi^{2}=0.3\right)$, 
even if more CMS students are planning to do a $\mathrm{PhD}$. The authors hypothesize that it may be still too early for an undergraduate student to project himself so far into the future.

- Regarding the bibliographic skills:

Here we checked whether the courses on bibliography taken by the CMS students are effective. We examined whether the students (whose mother tongue is French) are comfortable with bibliographic research and with reading articles in English.

\begin{tabular}{|c|c|c|c|c|}
\hline \multirow{3}{*}{$\begin{array}{l}\text { Statements for } \\
\text { Response }\end{array}$} & \multicolumn{4}{|c|}{ Students Answering Correctly } \\
\hline & \multicolumn{2}{|c|}{ CMS Group $a$} & \multicolumn{2}{|c|}{ Control Group } \\
\hline & $N$ & $\%$ & $N$ & $\%$ \\
\hline $\begin{array}{l}1 \text { Cite different } \\
\text { bibliographic } \\
\text { search portals. }\end{array}$ & 35 & 45 & 12 & 17 \\
\hline $\begin{array}{l}2 \text { Cite the names of } \\
\text { scientific journals } \\
\text { used in chemistry. }\end{array}$ & 21 & 27 & 7 & 10 \\
\hline $\begin{array}{l}3 \text { Provide an English } \\
\text { translation of the } \\
\text { following scientific } \\
\text { terms. }{ }^{c}\end{array}$ & 30 & 38 & 14 & 20 \\
\hline \multicolumn{5}{|c|}{$\begin{array}{l}a N=78 . b N=69 . \text {. The list of terms included words } \\
\text { such as "furnace", "beaker", "heating rate", "safety } \\
\text { glasses", etc. }\end{array}$} \\
\hline
\end{tabular}

The first question was to determine if students know the names of the databases, and the second one to see if they know the names of chemical journals. A third question asked if they can understand technical English words found in articles, such as furnace, beaker, heating rate, safety glasses etc. The students who have completed the module know of more search portals for bibliography $\left(\chi^{2}=12.7\right)$ and they know more scientific journals $\left(\chi^{2}=6.7\right)$. They are also able to translate more words from English to French $\left(\chi^{2}=5.7\right)$. All of these results concerning the skills related to bibliography are statistically significant.

- Regarding safety / security: 
The questions asked here indicate whether the information given on safety is profitable to CMS students. Questions about the meanings of pictograms in chemistry and safety rules were asked.

\begin{tabular}{|c|c|c|c|c|}
\hline \multirow{3}{*}{$\begin{array}{l}\text { Statements for } \\
\text { Response }\end{array}$} & \multicolumn{4}{|c|}{ Students Answering Correctly } \\
\hline & \multicolumn{2}{|c|}{ CMS Group $a$} & \multicolumn{2}{|c|}{ Control Group ${ }^{k}$} \\
\hline & $N$ & $\%$ & $N$ & $\%$ \\
\hline $\begin{array}{l}1 \text { Complete the } \\
\text { pictogram table. }\end{array}$ & 48 & 62 & 40 & 58 \\
\hline $\begin{array}{l}2 \text { What should you } \\
\text { do if a part of the } \\
\text { body comes into } \\
\text { contact with a } \\
\text { chemical product } \\
\text { such as } \\
\text { hydrochloric acid? }\end{array}$ & 78 & 100 & 65 & 94 \\
\hline$a_{N}=78 . b_{N}=69$ & & & & \\
\hline
\end{tabular}

Knowledge of pictograms is a little better for the "Chemistry: magical science" group (but the results are not statistically significant, $\chi^{2}=0.2$ ). Students who have taken the module react better to an accident $\left(\chi^{2}=4.6\right)$. These results show that the skills gained from studying this module seems to be effective and more deeply ingrained, even one or two years after the end of this training. The students who followed it are better able to answer questions related to bibliography and safety in chemistry than those who did not follow it.

Furthermore, at the end of their third university year, the students are involved in a tutored project where they must do a mini research project (including bibliographic research and experimental work). The grades for this project work for the students who have taken the CMS module seem better than for those who have not, whether one considers the average for the bibliographic research (average value 14.5/20 for the CMS versus 13/20 for the others), or the practical part (average value 15.5/20 for the CMS versus 14/20 for the others). According to the teachers, CMS students are more comfortable with this project as they have already carried out this type of work before, albeit on a smaller scale. 


\section{CONCLUSION}

This paper describes uses of an integrated learning environment based on a laboratory approach for a large variety of skills related to general chemistry course sequence. In this teaching course, experimentation, collaborative student involvement, lecture, technology and individual attention can be maximized for more effective learning.

Students receive a solid background in bibliographic skills and laboratory safety. The syntheses realized during the practical work lead to original materials, which have remarkable properties. Students also learn to write, to communicate their results both in writing and orally. Moreover, training for oral presentations is given in the module. The analyses of two different surveys point out the effect of this integrated module in terms of knowledge in laboratory safety, bibliography and in terms of positive feelings towards the CMS module. The results obtained from the analysis of surveys show that the population who have completed the module of "Chemistry: magical science" is more knowledgeable about these topics and thus more motivated towards future studies in the field of chemistry.

We hope that this kind of teaching can be more widely used, so that more students can have access to it in the future.

\section{ASSOCIATED CONTENT}

\section{ASSOCIATED CONTENT}

Supporting Information

The Supporting Information is available on the ACS Publications website at DOI:

10.1021/acs.jchemed.XXXXXXX. [ACS will fill this in.] Organization of the module. Protocols of the syntheses of the materials (ferrofluids, phosphorescent and ruby) (docx).

\section{AUTHOR INFORMATION}

\section{Corresponding Author}

*E-mail: karine.molvinger-verger@umontpellier.fr

\section{ACKNOWLEDGMENTS}

The authors thank the teaching staff who contribute to the effective running of this practical course: They also thank Naseem Ramsahye for proofreading the manuscript. 


\section{REFERENCES}

1. Bretz, S.L.; Fay, M; Bruck, L.B.; Towns, M.H. What Faculty Interviews Reveal about Meaningful Learning in the Undergraduate Chemistry Laboratory. J. Chem. Educ. 2013, 90, 281-288.

2. Obenland, C. A.; Kincaid K.; Hutchinson, J.S. A General Chemistry Laboratory Course Designed for Student Discussion. J. Chem. Educ. 2014, 91, 1446-1450.

3. Hawkes, S. J. Chemistry is not a Laboratory Science. J. Chem. Educ. 2004, 81, 1257.

4. Domin, D. S. A Review of Laboratory Instruction Styles. J. Chem. Educ. 1999, 76, 543-547.

5. Hofstein, A.; Lunetta, V.N. The Laboratory in Science Education: Foundations for the Twentyfirst Century. Sci. Educ. 2004, 88, 28-54.

6. Reid, N.; Shah, I., The Role of Laboratory Work in University Chemistry. Chemistry Education Research and Practice 2007, 8(2), 172-185.

7. Tiberghien, A.; Veillard, L.; Le Maréchal, J.F.; Buty, C.; Millar, R. An Analysis of Labwork Tasks Used in Science Teaching at Upper Secondary School and University Levels in Several European Countries. Sci. Educ. 2001, 85, 483-508.

8. Hofstein, A.; Mamlock-Naaman, R. The Laboratory in Science Education: the State of the Art. Chem. Educ. Res. Pract. 2007, 8, 105-107.

9. George-Williams, S.R.; Ziebell, A.L.; Kitson, R.R.A.; Coppo, P.; Thompson, C.D.; Overton T.L. 'What do you Think the Aims of Doing a Practical Chemistry Course are?' A Comparison of the Views of Students and Teaching Staff across three Universities. Chem. Educ. Res. Pract. 2018, 19, 463-473.

10. Furlan, P.Y.; Melcer, M.E. Removal of aromatic pollutant surrogate from water by recyclable magnetite-activated carbon nanocomposite: an experiment for general chemistry. J. Chem. Educ. 2014, 91, 1966-1970.

11. Porter, L.A. Chemical nanotechnology: a liberal arts approach to a basic course in emerging interdisciplinary science and technology. J.Chem.Educ. 2007, 84-2, 259-264.

12. Widstrand, C.G.; Nordell, K.J.; Ellis, A.B. Designing and reporting experiments in chemistry classes using examples from materials science: illustrations of the process and communication of scientific research. J.Chem.Educ. 2001, 78-8, 1044-1046.

13. Miller, K. M.; Hage, D. S. Survey of long-term integrated laboratory use in undergraduate chemistry programs. J. Chem. Educ. 1995, 72 (3), 248-250.

14. Budner, D.; Simpson, B. Project-Based Integrated Lecture and Laboratory Quantitative Analysis Course. J. Chem. Educ. 2018, 95, 1533-1540.

15. Monga, V.; Knox, K.J.; Gillis, E.A.L.; Stoodley, R.; Bussiere, G.; Rogers, C. Implementation of a Student-Customized Integrated Upper-Level Chemistry Laboratory Course. J. Chem. Educ. 2019, 96, 1609-1619.

16. A website is devoted to this module (http://www.chimiemagique.fr) (accessed Feb $3^{\text {rd }}, 2020$ ) 
17. Ashraf, S. S.; Marzouk, S.A.M.; Shehadi, I.A.; Murphy, B.M. An integrated Professional and Transferable Skills Course for Undergraduate Chemistry Students. J. Chem. Educ. 2011, 88, 4448.

18. Hubbard, B.A.; Jones, G.C.; Gallardo-Williams, M.T. Student-Generated Digital Tutorials in an Introductory Organic Chemistry Course. J. Chem. Educ. 2019, 96, 597-600.

19. Dalverny, A-L.; Leyral, G.; Rouessac, F.; Bernaud, L.; Filhol, J.-S. Synthesizing and Playing with Magnetic Nanoparticles: A Comprehensive Approach to Amazing Magnetic Materials. J. Chem. Educ. 2018, 95, 121-125.

20. Filhol, J. S.; Zitoun, D.; Bernaud, L.; Manteghetti, A. Microwave Synthesis of a Long-Lasting Phosphor. J. Chem. Educ. 2009, 89, 72-75.

21. Leyral, G.; Bernaud, L.; Manteghetti, A.; Filhol, J. S. Microwave Synthesis of a Fluorescent Ruby Powder. J. Chem. Educ. 2013, 90, 1380-1383.

22. Berger, P.; Adelman, N.B.; Beckman,K.J.; Campbell, D.J.; Ellis, A.B.; Lisensky, G.C. Preparation and properties of an aqueous ferrofluid. J. Chem. Educ. 1999, 76-7, 943-948.

23. Bentley, A.K.; Farhoud, M.; Ellis, A.B.; Lisensky, G.C.; Nickel, A-M. L.; Crone, W.C. Template synthesis and magnetic manipulation of nickel nanowires. J. Chem. Educ. 2005, 82-5, 765-768.

24. Furlan, P.Y.; Fisher, A.J., Melcer, M.E.; Furlan, A.Y.; Warren, J.B. Preparing and testing a magnetic antimicrobial silver nanocomposite for water disinfection to gain experience at the nanochemistry-microbiology interface. J. Chem. Educ. 2017, 94, 488-493.

25. Winkelmann, K.; Noviello, T.; Brooks, S. Preparation of CdS nanoparticles by first-year undergraduates. J. Chem. Educ. 2007, 84-4, 709-710.

26. Boatman, E.M.; Lisensky, G.C.; Nordell, K.J. A safer, easier, faster synthesis for CdSe quantum dot nanocrystals. J. Chem. Educ. 2005, 82-11, 1697-1699. 